NEC severity and the influence of enteral nutrition in a Caucasian population compared to a historic control group.

Material and methods Since 2010, VLBW infants born

Results 230 infants were included (mean gestational age: $27+2$, birth weight: 900g) and compared with 233 controls $(28+5$, $980 \mathrm{~g})$. After implementation of Infloran ${ }^{\circledR}$ NEC decreased by $32 \%$ (10.3\% before vs. $7 \%$ after implementation of probiotics, $\mathrm{p}$ $=0.092-$ corrected for confounding variables birth weight and gestational age). Probiotics had no influence on NEC severity. A NEC reduction was shown in breast fed infants only and not in formula fed infants.

Discussion The effect of Infloran ${ }^{\circledR}$ was less effective in our Caucasian population than expected. Interestingly, NEC incidence was not reduced in exclusively formula fed infants. The inefficacy in this subgroup is alarming. Therefore, the impact of enteral nutrition on probiotic effects should be explored in further prospective randomised controlled trials.

\section{PS-182 EFFECT OF COMBINED USAGE OF PREBIOTIC OLIGOSACCHARIDES ON THE GROWTH OF BIFIDOBACTERIUM BREVE}

T Ehara, H Izumi, M Tsuda, H Iwamoto, K Namba, Y Takeda. Nutritional Science Institute, Morinaga Milk Industry Co. Ltd., Zama, Japan

10.1136/archdischild-2014-307384.479

Non-digestive oligosaccharides are often added to infant formula to help formula-fed infants develop an intestinal microbiota composed predominantly of Bifidobacteria, similar to that of breastfed infants. Because various types of oligosaccharides exhibit specific microbial metabolism, the combined usage of oligosaccharides is considered to provide additive or synergistic effects for Bifidobacteria growth in the intestinal microbiota. The aim of this study was to evaluate the combined effect of lactulose, raffinose, and galacto-oligosaccharide (GOS) on the growth of Bifidobacterium breve, one of the major Bifidobacteria found in infant intestinal microbiota, using an in vitro mixed culture model. Seven typical bacterial species found in infant intestinal microbiota, including $B$. breve, were selected and then co-cultured under anaerobic conditions to mimic the infant intestinal environment. Each oligosaccharide was added to the medium, alone or in combination with other oligosaccharides. At all times, the total amount of added oligosaccharides composed $1 \%$ of the medium. Cells were harvested after several hours of incubation, and bacterial genomic DNA was extracted. Bacterial cell numbers were determined using quantitative realtime-PCR, with specific primers targeting the $16 \mathrm{~s}$ rRNA genes of different bacterial groups. The combination of lactulose, raffinose, and GOS promoted the growth of Bifidobacterium breve compared with any single oligosaccharide or the combination of lactulose and raffinose. The combined usage of lactulose, raffinose, and GOS may provide the benefit of promoting a Bifidobacteria-predominant intestinal microbiota in formulafed infants.

\section{PS-183 BLOOD TRANSFUSIONS ARE NOT A RISK FACTOR FOR NECROTIZING ENTEROCOLITIS IN EXTREMELY PRETERM INFANTS}

L Larsson, P Challis, C Späth, E Stoltz Sjöström, M Domellöf. Department of Clinical Sciences, Pediatrics Umeå University Sweden, Umeå, Sweden

10.1136/archdischild-2014-307384.480
Background Transfusion practices are highly variable between hospitals and previous studies have suggested that blood transfusions may increase the risk of necrotizing enterocolitis (NEC).

Aim To explore the association between blood transfusions and incidence of NEC in extremely preterm infants.

Methods We used data from a Swedish population-based study including extremely preterm infants $(<27$ weeks) born between 2004-2007, ( $\mathrm{n}=602$ ). All data on blood transfusions and haemoglobin $(\mathrm{Hb})$ concentrations up to 28 days of age was collected for survivors. We performed a nested case-control study where two controls were chosen for each case of NEC $(n=21)$. Results During the first 28 days of life, infants received a median $\left(25^{\text {th }}-75^{\text {th }}\right.$ percentile) of 6 (3-9) blood transfusions resulting in $75(44-120) \mathrm{ml} / \mathrm{kg}$ of blood. Predictors for receiving a higher volume of blood transfusions were days on respiratory support $(\mathrm{R}=0.345, \mathrm{p}<0.001)$, hospital $(\mathrm{R}=0.339, \mathrm{p}<$ $0.001)$, low birth weight $(\mathrm{R}=-0.236, \mathrm{p}<0.001)$ and total steroid dose $(\mathrm{R}=0.209, \mathrm{p}<0.001)$. Hb was not a significant predictor.

Overall NEC incidence was 5.8\%. There was no significant difference between NEC cases and controls in number of blood transfusions $(p=0.420)$, volume of blood transfused from birth to NEC diagnosis $(\mathrm{p}=0.274)$, or during the $48 \mathrm{~h}$ preceding NEC diagnosis $(\mathrm{p}=0.459)$.

Conclusions Blood transfusions were given liberally in Sweden compared to other studied populations. Morbidity related variables, especially those related to respiratory illness, were significant predictors of blood transfusion. NEC incidence was comparable with other populations but no significant association was found between blood transfusions and NEC among these extremely preterm infants.

\section{PS-184 INTESTINAL PERMEABILITY PRECEDING NECROTISING ENTEROCOLITIS AND SEPSIS IN PRETERM INFANTS}

${ }^{1}$ PF Fleming, ${ }^{2} \mathrm{~S}$ Eaton, ${ }^{3} \mathrm{M}$ Wilks, ${ }^{3} \mathrm{MR}$ Millar, ${ }^{1} \mathrm{KL}$ Costeloe. ${ }^{1}$ Paediatrics, Queen Mary University of London, London, UK; ${ }^{2}$ Institute of Child Health, University College London, London, UK; ${ }^{3}$ Microbiology, Barts Health NHS Trust, London, UK

\subsection{6/archdischild-2014-307384.481}

Introduction Increased intestinal permeability may precede the onset of several important diseases in preterm infants including necrotising enterocolitis (NEC) and Gram negative septicaemias.

Hypothesis that increased intestinal permeability is evident at 2 weeks of age and may precede the onset of NEC or Gram negative septicaemias.

Methods Infants $<31$ weeks gestation were enrolled. Intestinal permeability was assessed by the sugar absorption test (SAT) using lactulose and mannitol and gut leakage by stool alpha-1antitrypsin (A1AT). Clinical data were prospectively collected.

Results Thirty-six infants were enrolled. The median (range) gestation was 27 weeks (24-30) and median birth weight was $900 \mathrm{~g}(585-1460)$. Nine infants (25\%) developed suspected or proven NEC (any NEC) of whom 5 (14\%) developed $\geq$ Bells Stage II NEC. Four infants (11\%) developed Gram negative septicaemias. Results are compared between infants with either NEC or sepsis and those with neither.

The median (range) lactulose:mannitol ratio (L:M) for all infants was $0.38(0.01-5.46)$ and median A1AT was 128 (41$1518) \mathrm{mg} / \mathrm{L}$. There was no statistically significant difference by $\mathrm{L}: \mathrm{M}$ in infants who developed any NEC ( $\mathrm{p}=0.75) ; \geq$ Bells 
Stage II NEC $(p=0.82)$ or sepsis $(p=0.21)$ nor of stool alpha1 -antitrypsin in those with any NEC $(p=0.70)$; $\geq$ Bells Stage II NEC $(p=0.87)$ or sepsis $(p=0.81)$.

Discussion In this cohort, the SAT by lactulose:mannitol ratios and stool A1AT did not show evidence of increased intestinal permeability at 2 weeks of age in infants who subsequently developed NEC or sepsis.

\section{PS-185 INTESTINAL OXYGEN EXTRACTION STRONGLY CORRELATES WITH I-FABP LEVELS, A MARKER FOR INTESTINAL DAMAGE}

${ }^{1}$ TE Schat, ${ }^{2} \mathrm{FH}$ Heida, ${ }^{2} \mathrm{M}$ Schurink, ${ }^{1} \mathrm{CV}$ Hulzebos, ${ }^{1}$ AF Bos, ${ }^{1} \mathrm{EMW}$ Kooi, ${ }^{2} \mathrm{JBF}$ Hulscher. ${ }^{1}$ Division of Neonatology, Beatrix Children's Hospital/University Medical Center Groningen, Groningen, Netherlands; 'Division of Pediatric Surgery, Department of Surgery/University Medical Center Groningen, Groningen, Netherlands

\subsection{6/archdischild-2014-307384.482}

Background and aim It remains unknown whether near-infrared spectroscopy (NIRS) can be used to assess intestinal perfusion. Intestinal fatty acid binding proteins in plasma (I-FABPp) and urine $(\mathrm{I}-\mathrm{FABPu})$ are a direct measure of intestinal epithelial cell damage that may occur after intestinal hypoperfusion. We measured splanchnic fractional tissue oxygen extraction (FTOE) and correlated these FTOE values with I-FABP levels in preterm infants in the first $16 \mathrm{~h}$ after onset of necrotizing enterocolitis (NEC).

Methods Preterm infants born between October 2010 and November 2012 were prospectively included when NEC was diagnosed (Bell stage $\geq 2$ ). Regional tissue oxygen saturation of the liver $\left(\mathrm{r}_{\text {liv }} \mathrm{SO} 2\right)$ and infra-umbilical $\left(\mathrm{r}_{\text {int }} \mathrm{SO} 2\right)$ region were measured continuously by NIRS. Mean 8-hour FTOE values were calculated: FTOE $=\left(\mathrm{SpO}_{2}-\mathrm{rSO}_{2}\right) / \mathrm{SpO}_{2}$. Plasma and urine samples collected in the first $16 \mathrm{~h}$ after onset of symptoms were

Abstract PS-185 Table 1 Correlation coefficients between FTOE and I-FABP

\begin{tabular}{|ll|l|}
\hline 0-8 hours & & \\
\hline & I-FABPp & I-FABPu \\
\hline livFTOE & $\rho=0.600$ & $\rho=0.500$ \\
& $P=0.285$ & $P=0.667$ \\
& $\mathrm{n}=5$ & $\mathrm{n}=3$ \\
\hline intFTOE & $\rho=0.900$ & $\rho=1.000$ \\
& $P=0.037$ & $P=<0.001$ \\
& $\mathrm{n}=5$ & $\mathrm{n}=3$ \\
\hline 8-16 hours & & \\
\hline & I-FABPp & I-FABPu \\
\hline livFTOE & $\rho=0.881$ & $\rho=0.679$ \\
& $P=0.004$ & $P=0.094$ \\
& $\mathrm{n}=8$ & $\mathrm{n}=7$ \\
\hline intFTOE & $\rho=0.745$ & $\rho=0.833$ \\
& $P=0.013$ & $P=0.005$ \\
& $\mathrm{n}=10$ & $\mathrm{n}=9$ \\
\hline
\end{tabular}

used for analysis. Spearman's correlation test was used to calculate correlation coefficients.

Results Twenty-one preterm infants were included (median [range] gestational age 28 [25-36] weeks, birth weight 1290 [740-2400] grams). Median [range] liver FTOE (livFTOE) was 0.33 [0.07-0.81], infra-umbilical FTOE (intFTOE) 0.48 [0.13$0.82]$, I-FABPp $16.3[0.54-3748] \mathrm{ng} / \mathrm{mL}$, and I-FABPu 89.9 [3.2-23,336] ng/mL. Table 1 shows strong positive correlations between FTOE and I-FABP levels.

Conclusion High intFTOE values, suggestive of an impaired intestinal blood flow, correlated strongly with I-FABP, i.e. with the extent of intestinal epithelial cell damage. These results indicate that intestinal NIRS monitoring can be used to assess intestinal perfusion in preterm infants with an impaired intestinal blood flow such as occurs in NEC.

\section{PS-186 BLOOD LACTATE AS A PREDICTIVE MARKER FOR NEONATAL NECROTISING ENTEROCOLITIS (NEC) SEVERITY AND OPERATIVE OUTCOMES}

AK Davenport, S Chuang, P Desai. Neonatal Unit, Chelsea and Westminster NHS Foundation Trust, London, UK

\subsection{6/archdischild-2014-307384.483}

Background and aims Lactate as a marker for tissue perfusion and hypoxia is increasingly used in routine point-of-care monitoring in critical care. We investigated the association of preoperative lactate (PreL) with operative outcomes and mortality in neonatal surgical NEC.

Methods 25 infants with NEC confirmed on laparotomy at a tertiary surgical centre were retrospectively evaluated. Maximal PreL was categorised as normal $(<2 \mathrm{mmol} / \mathrm{L})$, moderate $(2-5$ $\mathrm{mmol} / \mathrm{L})$ or severe $(>5 \mathrm{mmol} / \mathrm{L})$, and correlated to extent of NEC involvement and mortality.

Results Median birth gestation and weight were 27weeks (range 23-33) and 1035g (555-2060). Median PreL was 5.1(range 0.616.2) $\mathrm{mmol} / \mathrm{L}$. Elevated PreL correlated with NEC severity, with predominance of pan-intestinal and multifocal involvement in severe hyperlactaemia (Table 1). Infants with isolated NEC were observed to have normal or moderate-PreL. Moderate to severe PreL was also associated with increased mortality rate and need for further surgery.

Conclusions This preliminary study suggests that pre-operative hyperlactaemia and degree of elevation may be associated with a poor prognosis in infants with surgical NEC. Further larger studies may enable better evaluation of its use an adjunctive monitoring or prognostic tool in guiding early neonatal NEC management.

Abstract PS-186 Table 1 Surgical outcomes and Preoperative lactate (PreL)

\begin{tabular}{llll}
\hline & $\begin{array}{l}\text { Normal-PreL } \\
(<2 \mathrm{mmol} / \mathrm{L}) \\
(\mathrm{n}=3)\end{array}$ & $\begin{array}{l}\text { Moderate-PreL } \\
(2-5 \mathrm{mmol} / \mathrm{L})\end{array}$ & $\begin{array}{l}\text { Severe-PreL } \\
(\mathrm{n}=5 \mathrm{mmolL})\end{array}$ \\
\hline Pan-NEC $(\mathrm{n}=13)$ & $0(0 \%)$ & $3(33.3 \%)$ & $10(76.9 \%)$ \\
Multifocal-NEC $(\mathrm{n}=6)$ & $1(33.3 \%)$ & $2(22.2 \%)$ & $3(23.1 \%)$ \\
Isolated-NEC $(\mathrm{n}=6)$ & $2(66.7 \%)$ & $4(44.4 \%)$ & $0(0 \%)$ \\
Further surgery* $(\mathrm{n}=7)$ & $0(0 \%)$ & $2(22.2 \%)$ & $5(38.5 \%)$ \\
Mortality $(\mathrm{n}=6)$ & $0(0 \%)$ & $2(22.2 \%)$ & $4(30.8 \%)$ \\
\hline
\end{tabular}

